\title{
Hepatic angiomyolipoma presenting with chronic epigastric pain: A case report
}

\author{
Anthony Relucio Perez, Clarence Yacapin
}

\begin{abstract}
Introduction: Angiomyolipomas primarily arising from the liver are rare. Hepatic angiomyolipoma (HAML) is a rare tumor characterized by the presence of blood vessels, muscle tissue and adipose tissue with only about 300 reported cases to date. This is the first case reported in literature of a Filipino patient. Case Report: A 49-year-old Filipino female presented with a two-year history of recurrent epigastric pain. Abdominal magnetic resonance imaging revealed a fat-containing lesion in the left liver lobe. Serial follow-up revealed progressive enlargement on imaging studies. Serologic tests were negative for malignancy or infection. The patient underwent left hepatic lateral segmentectomy which revealed a $7.5 \times 7 \mathrm{~cm}$ friable mass involving hepatic segments II and III. Histopathology showed mature fat cells with a few thick-walled blood vessels and spindled smooth muscle cells with no atypia. Homatropine methylbromide-45 test showed strong and diffused staining confirming angiomyolipoma. Conclusion: Clinical evaluation and a comprehensive analysis of ultrasonography,
\end{abstract}

Anthony Relucio Perez ${ }^{1}$, Clarence Yacapin ${ }^{2}$

Affiliations: ${ }^{1} \mathrm{MD}, \mathrm{MHA}$, Vice Chair for Training, Department of Surgery, UP College of Medicine and Philippine General Hospital, Section Chief, Hepatobiliary and Pancreatic Surgery Asian Hospital and Medical Center; ${ }^{2} \mathrm{MD}$, Clinical Fellow Section of Minimally Invasive Surgery, Department of Surgery Asian Hospital and Medical Center.

Corresponding Author: Anthony Relucio Perez, 108 Dayap Street Ayala Alabang, Muntinlupa, NCR, Philippines, 1780; Email: tonyperez92@yahoo.com

Received: 26 June 2017

Accepted: 26 July 2017

Published: 21 August 2017 computed tomography scan and magnetic resonance imaging scan are essential for correct preoperative diagnosis. Symptoms, malignant potential and inability to distinguish from liver malignancies may indicate surgical resection as the best treatment option.

Keywords: Angiomyolipoma, Hepatic mass, Liver tumors

\section{How to cite this article}

Perez AR, Yacapin C. Hepatic angiomyolipoma presenting with chronic epigastric pain: A case report. Int J Hepatobiliary Pancreat Dis 2017;7:28-31.

Article ID: 100070IJHPDAP2017

$* * * * * * * * *$

doi: 10.5348/ijhpd-2017-70-CR-6

\section{INTRODUCTION}

Hepatic angiomyolipoma (HAML) is a rare tumor characterized by the presence of blood vessels, muscle tissue and adipose tissue. It was first described by Ishak in 1976 and was classified under the group of perivascular epithelioid tumors in the World Health Organization classification of tumors in 2002 [1, 2]. Approximately 300 reported cases have been reported to date and to the best of our knowledge, this is the first reported case of HAML in a Filipino patient.

Hepatic angiomyolipoma is difficult to diagnose because of its rarity and varying composition of adipose and muscle tissue. Tsui et al. categorized the disease according to its composition: mixed, lipomatous ( $>70 \%$ fat), myomatous ( $<10 \%$ fat) and angiomatous types 
[3]. These varying classifications resembles other hepatic tumors, and makes preoperative diagnosis only $11-50 \%$ accurate, where majority are misdiagnosed as hepatocellular carcinoma (HCC) [3-5].

While HAML is considered a benign tumor, several malignancies and recurrences have been reported. Predisposing characteristics of its invasive nature have been unclear. It is important to review the characteristics that improve accuracy in the diagnosis of HAML as well as the features that might predispose to invasiveness.

\section{CASE REPORT}

A 49-year-old Filipino female presented with a twoyear history of liver mass with recurrent epigastric pain. She was being managed as a case of gastritis with a benign liver mass for two years until she consulted at our institution for increasing severity and recurrence of epigastric pain. There was no abdominal tenderness no palpable mass noted on physical examination. Abdominal magnetic resonance imaging (MRI) with intravenous gadolinium contrast revealed a fat-containing lobulated lesion in the left liver lobe with increasing size measuring $7.12 \times 6.2 \mathrm{~cm}$ compared to $6.15 \times 5.17 \mathrm{~cm}$ from the previous imaging study done two years prior (Figure 1). Serologic tests showed no indication of viral hepatitis infection and normal alpha fetoprotein and carcinoembryonic antigen.

The patient was presented with treatment options and eventually requested surgical resection. Routine preoperative testing revealed no significant comorbidities. Intraoperative findings confirmed the location of the mass and a lipomatous appearance of the liver lesion (Figure 2). She underwent left hepatic lateral segmentectomy with unremarkable perioperative course. The gross specimen showed a $7.5 \times 7 \mathrm{~cm}$ spongy, friable and fatty yellowish mass involving hepatic segments II and III (Figure 3). Histopathology revealed a non-encapsulated mass composed mainly of mature fat cells with a few scattered islands of thick-walled blood vessels and spindled smooth muscle cells with no atypia (Figure 4). Homatropine mehtylbromide-45 (HMB45) test showed strong and diffused staining of the specimen which confirmed the diagnosis of angiomyolipoma (Figure 5). After one year of follow-up, the patient did not show any recurrence or postoperative complications.

\section{DISCUSSION}

Hepatic angiomyolipoma is a benign mesenchymal tumor, often mistaken for hepatocellular carcinoma (HCC) preoperatively because of its similarity in its diagnostic imaging characteristics. Improving knowledge of clinical features and imaging characteristics will help decrease misdiagnosis and overtreatment.

A large case series of 33 HAML patients revealed that majority occur in women with a peak incidence age range

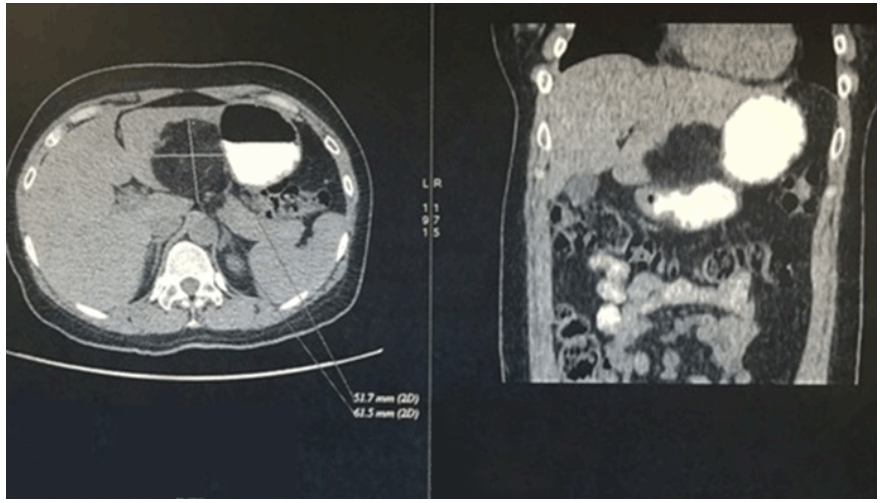

Figure 1: Magnetic resonance imaging scan with gadolinium contrast.

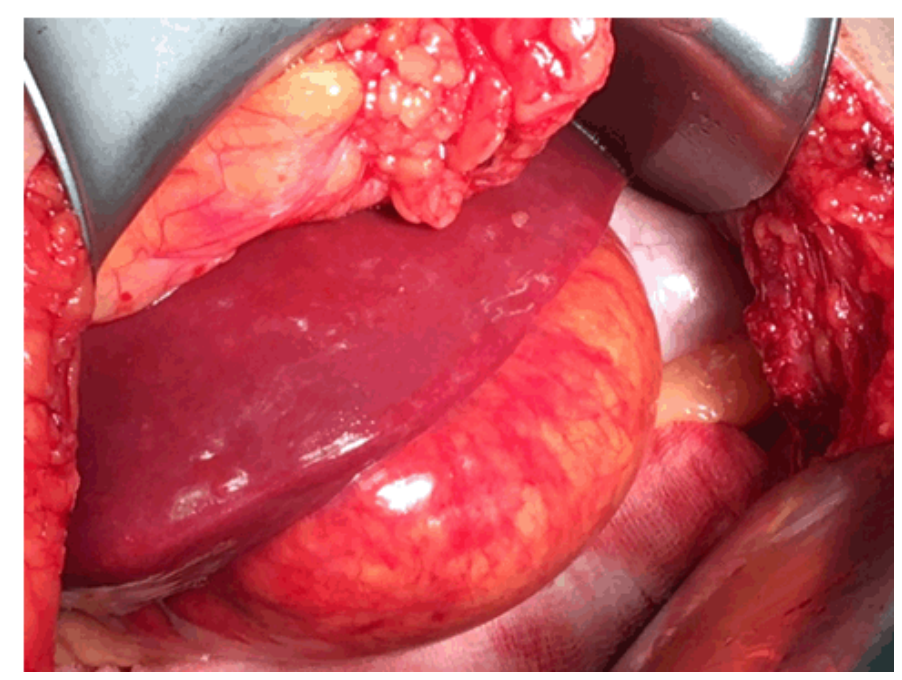

Figure 2: Intraoperative findings.

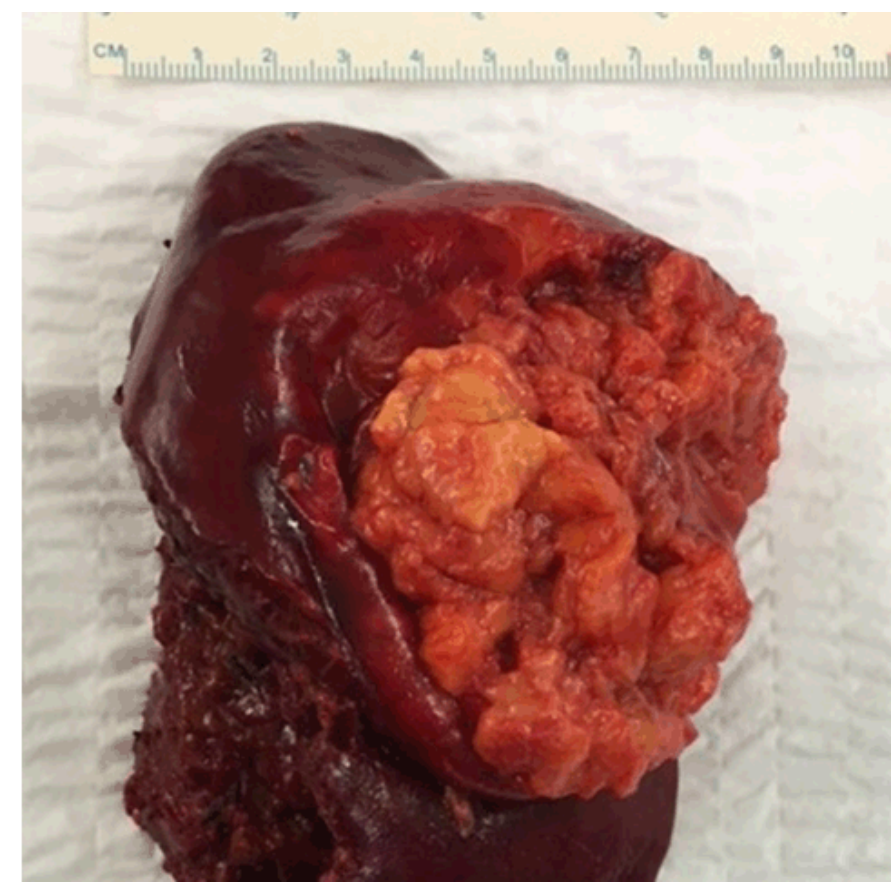

Figure 3: Resected specimen. 


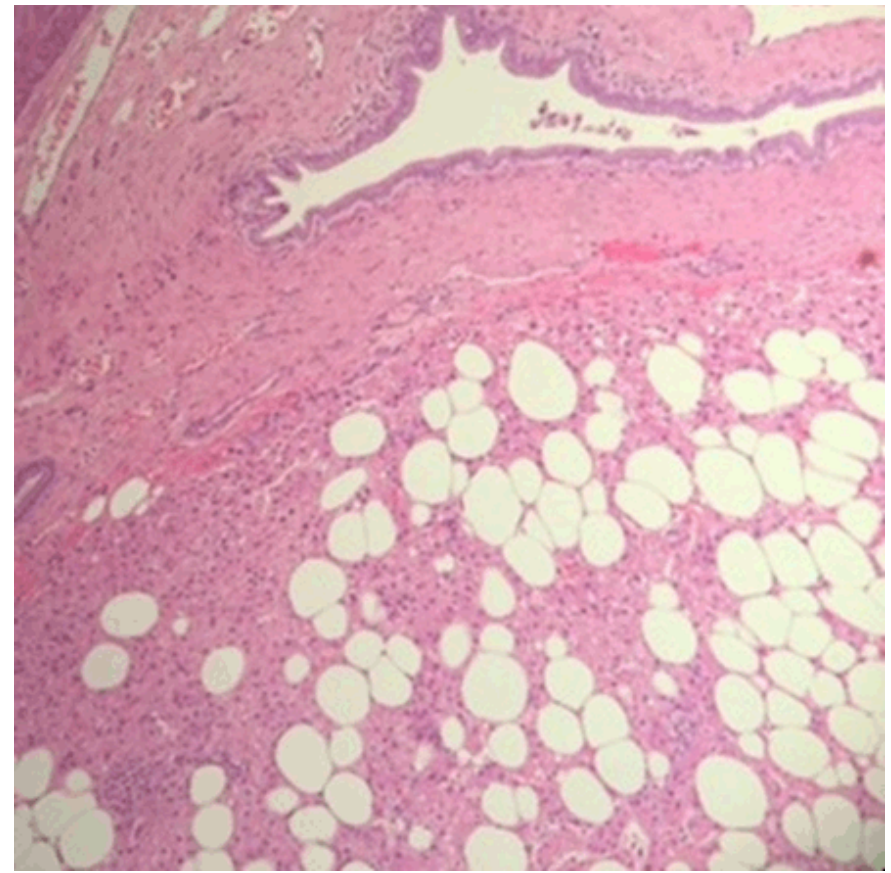

Figure 4: Histopathology showing adipose cells, muscle cells and blood vessel (H\&E stain, x100).

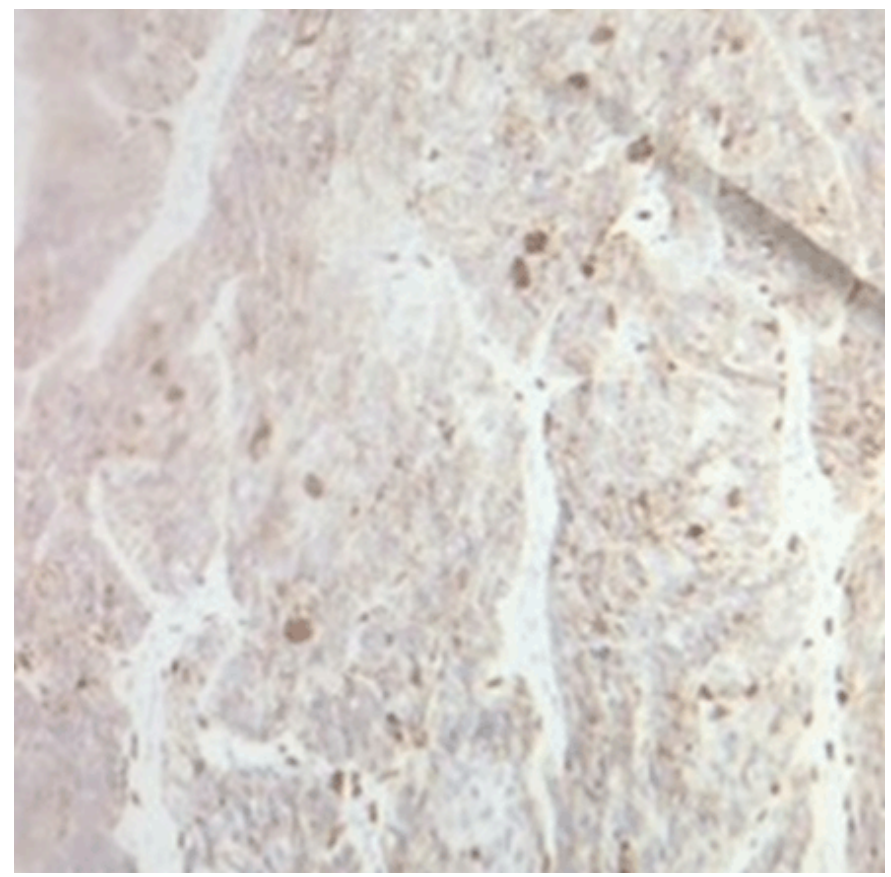

Figure 5: Homatropine mehtylbromide-45 (HMB45) test showing strong and diffused staining (magnification: $\mathrm{x400).}$

of $30-50$ years, and with $66 \%$ of the cases coming from Asian countries while $23 \%$ come from European countries [6]. Most patients are asymptomatic and diagnosed incidentally. Symptomatic patients present with right upper abdominal pain, abdominal distention, fever, discomfort and weight loss [4, 7]. Although symptoms are not always correlated with size, gastrointestinal symptoms as a result of intralesional bleeding are generally observed with tumors $>5.0 \mathrm{~cm}[6,8]$.

Differences in imaging features of HAML and HCC are important to avoid misdiagnosis and overtreatment. Zhu et al. compared imaging findings of 14 cases with existing literature and noted that HAML usually have an early draining vein in the arterial phase and tortuous vessels in the tumor. These features are absent in patients with HCC [9]. Furthermore, tumor capsule, liver cirrhosis, bile duct dilatation and lymphadenopathy, which are usually present in HCC, are absent in HAML [9]. These distinguishing features significantly contributed in considering angiomyolipoma in our patient preoperatively.

Although HAML is considered a benign tumor, several cases of have been recurrence or malignancy reported. Dalle et al. reported the first case of HAML with malignant vessel invasion that recurred five months after surgical resection [10]. Definite factors predisposing to malignancy is still unknown. However, a tumor size of $10 \mathrm{~cm}$ or greater seems to have greater risk of recurrence [11]. Although the occurrence of malignancy is very rare among patients with HAML, it is still important to advise close follow-up particularly among patients with large tumors.

\section{CONCLUSION}

Hepatic angiomyolipoma is a rare and benign entity. It has a wide variation and poses a diagnostic challenge clinically, radiologically, and pathologically. Its prognosis is good regardless of management, including hepatic resection or conservative treatment after tissue biopsy by echo-guided needle. Reports of aggressive growth patterns, malignant degeneration, recurrences and difficulty in definitive diagnosis mandates individualized treatment planning. As in this case, surgery may be the most appropriate treatment for symptomatic and progressively enlarging HAML. This case report adds to the pool of information on hepatic angiomyolipoma and hopefully raises awareness of the disease among Filipino surgeons. Improving knowledge by continuously updating and comparing characteristics of angiomyolipoma cases with existing literature can provide better understanding, diagnosis and effective care for patients.

$* * * * * * * * *$

\section{Author Contributions}

Anthony Relucio Perez - Substantial contributions to conception and design, Acquisition of data, Analysis and interpretation of data, Drafting the article, Revising it critically for important intellectual content, Final approval of the version to be published

Clarence Yacapin - Analysis and interpretation of data, Revising it critically for important intellectual content, Final approval of the version to be published 


\section{Guarantor}

The corresponding author is the guarantor of submission.

\section{Conflict of Interest}

Authors declare no conflict of interest.

\section{Copyright}

(C) 2017 Anthony Relucio Perez et al. This article is distributed under the terms of Creative Commons Attribution License which permits unrestricted use, distribution and reproduction in any medium provided the original author(s) and original publisher are properly credited. Please see the copyright policy on the journal website for more information.

\section{REFERENCES}

1. Ishak KG. Mesenchymal tumors of the liver. In: Okuda K, Peter KL. editors. Hepatocellular Carcinoma. New York: John Wiley \& Sons; 1976. p. 247-307.

2. Bonetti F, Pea M, Martignoni G, Zamboni G. PEC and sugar. Am J Surg Pathol 1992 Mar;16(3):307-8.

3. Tsui WM, Colombari R, Portmann BC, et al. Hepatic angiomyolipoma: A clinicopathologic study of 30 cases and delineation of unusual morphologic variants. Am J Surg Pathol 1999 Jan;23(1):34-48.
4. Zhou YM, Li B, Xu F, Wang B, et al. Clinical features of hepatic angiomyolipoma. Hepatobiliary Pancreat Dis Int 2008 Jun;7(3):284-7.

5. Hu WG, Lai EC, Liu H, et al. Diagnostic difficulties and treatment strategy of hepatic angiomyolipoma. Asian J Surg 2011 Oct;34(4):158-62.

6. Maebayashi T, Abe K, Aizawa T, et al. Improving recognition of hepatic perivascular epithelioid cell tumor: Case report and literature review. World J Gastroenterol 2015 May 7;21(17):5432-41.

7. Yang CY, Ho MC, Jeng YM, Hu RH, Wu YM, Lee PH. Management of hepatic angiomyolipoma. J Gastrointest Surg 2007 Apr;11(4):452-7.

8. Pedrassa BC, da Rocha EL, Kierszenbaum ML, Bormann RL, Torres LR, D’Ippolito G. Uncommon hepatic tumors: Iconographic essay - Part 1. Radiol Bras 2014 Sep-Oct;47(5):310-6.

9. Zhu Z, Yang L, Zhao XM, Luo DQ, Zhang HT, Zhou CW. Myomatous hepatic angiomyolipoma: Imaging findings in 14 cases with radiological-pathological correlation and review of the literature. Br J Radiol 2014 Jun;87(1038):20130712.

10. Dalle I, Sciot R, de Vos R, et al. Malignant angiomyolipoma of the liver: A hitherto unreported variant. Histopathology 2000 May;36(5):443-50.

11. Kamimura $\mathrm{K}$, Nomoto M, Aoyagi Y. Hepatic angiomyolipoma: Diagnostic findings and management. Int J Hepatol 2012;2012:410781.
Access full text article on other devices

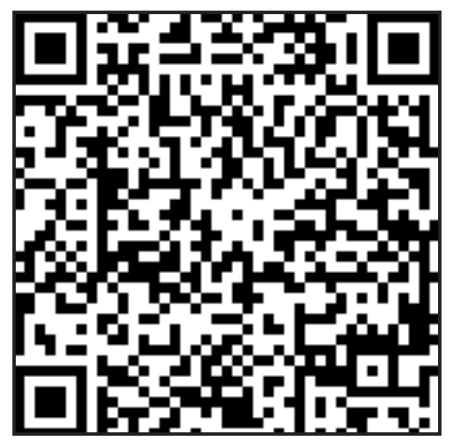

Access PDF of article on other devices

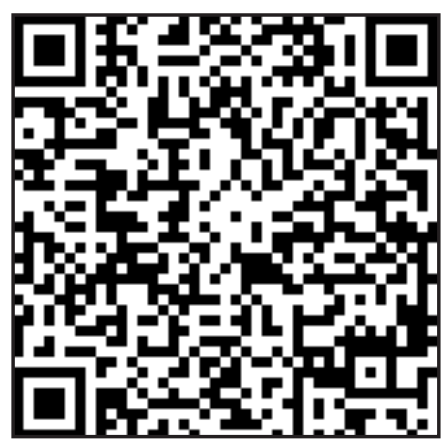

